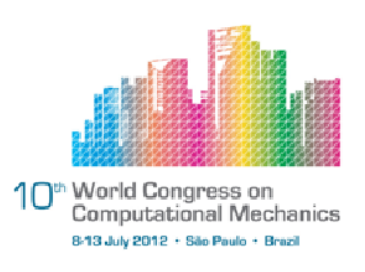

\title{
THERMO-FLUID-DYNAMICS QUENCHING MODEL: EFFECT ON MATERIAL PROPERTIES
}

\author{
D. N. Passarella ${ }^{1 *}$, R. L-Cancelos ${ }^{2}$, I. Vieitez ${ }^{1}$, F. $\operatorname{Varas}^{1,3}$, E. B. Martín ${ }^{4}$ \\ ${ }^{1}$ Departamento de Matemática Aplicada II, E. de Ing. de Telecomunicación, Universidad de \\ Vigo, Campus Marcosende, 36310 Vigo, España.*(diego@dma.uvigo.es) \\ ${ }^{2}$ Departamento de Ingeniería de los Materiales, Mecánica Aplicada y Construcción, E. de \\ Ing. Industrial, Universidad de Vigo, Campus Marcosende, 36310 Vigo, España \\ ${ }^{3}$ Departamento de Fundamentos Matemáticos. E.T.S. de Ing. Aeronáuticos, Universidad \\ Politécnica de Madrid, Pl. Cardenal Cisneros 3, 28040 Madrid, España \\ ${ }^{4}$ Departamento de Ingenería Mecánica, Máquinas y Motores Térmicos y Fluidos, E. de Ing. \\ Industrial, Universidad de Vigo, Campus Marcosende, 36310 Vigo, España
}

\begin{abstract}
A fully-coupled model of quenching by submerging for steel workpieces is presented. The model includes cooling of the piece due to piece-to-bath heat transfer calculations by solving the multiphase problem of an evaporable fluid, as well as the corresponding metallurgical transformations, the generation of residual stresses and associated geometrical distortions. The heat transfer model takes into account different boiling stages, from film boiling at very high workpiece surface temperatures, to single-phase convection at surface temperatures below saturation. The evolution and activation of each heat transfer mechanism depend on the dynamics of the vapor-liquid multiphase system of the quenching bath. The multiphase flow was modeled using the drift-flux mixture model, including an equation of conservation of energy of the liquid phase. Metallurgical transformations, geometrical distortions and residual stresses at the end of the process, are obtained based on the different cooling rates along the piece. The final distribution of metallurgical phases is obtained by the integration of the thermal evolution and using information of the CCT diagrams of studied steels. The analysis of deformations and residual stresses takes into account elasto-plasticity (without viscosity effects), transformation induced plasticity and hardening restoring phenomena. Comparison of results considering the approach presented here versus a simplified heat transfer model indicates that the level of induced residual stresses are noticeable different implying the necessity of developing a more precise heat transfer quenching model.
\end{abstract}

Keywords: Steel Heat Treatment, Quenching, Two-Phase Flow, Heat Partition Model, Residual Stresses 


\section{INTRODUCTION}

Quenching is a technological process that involves cooling of a heated workpiece in order to improve its mechanical properties. Undesired effects, such as geometrical distortions and/or generation of large residual stresses, can also be developed during it.

As any other process of technological interest, several efforts were made in order to describe it through mathematical models. In this area it is important to remark that modeling of steel quenching can be divided in at least three sub-problems. One major problem is the modeling of metallurgical transformations. This part is well known and the available models are robust and reliable [1,2]. On the other hand, we have the generation of residual stresses and geometrical distortions. These complex phenomena are also well covered and refined models can be found in specialized literature $[3,4,5,6]$. Finally, these two sub-problems strongly rely on an accurate description of the temperature evolution inside the piece. The thermal problem is usually solved taken a very rough description of heat exchanged to the cooling media $[7,8,9]$. The most precise models usually consider just a heat transfer coefficient dependent on wall temperature $\left(h\left(T_{w}\right)\right)$ to describe all the stages of cooling. This approach relies on experimental data obtained from configurations different to the modeled ones (for instance, a Jominy test, [7, 8, 9]) and is completely independent on local flow conditions such as flow velocity, bath temperature and vapor fraction. Improvement of current techniques was performed in [10] where the effect of different boiling modes is incorporated by means of a set of correlations formulated for water.

It is important to remark that all the complexity of metallurgical and mechanical models is not exploited and the accuracy of its results hampered if the thermal problem is solved using an approach as simple as the previously mentioned. In this work, the simulation of quenching in oil is developed based on a novel heat transfer model previously developed by the authors [11]. Evolution of phases distribution and stresses is obtained along the process for two different steels. A medium alloy steel (approx. $0.16 \% \mathrm{C}, 0.74 \% \mathrm{Ni}, 0.18 \% \mathrm{Cr}, 0.48 \% \mathrm{Mo}$, $1.30 \% \mathrm{Mn}$ ) and an eutectoid one (approx. $0.77 \% \mathrm{C}$ ) are selected in order to obtain examples of different distribution of metallurgical phases at the end of the process. Competition between stresses of thermal origin and the ones due to metallurgical transformations will dictate the final stress state on the workpiece. Comparison of results obtained with our model versus a simplified model based on a heat transfer coefficient only dependent on wall temperature $\left(h\left(T_{w}\right)\right)$ is presented.

\section{MODEL}

As was previously mentioned, quenching process and its effects on the treated workpieces, can be regarded as several sub-problems that have to be tackled by specific models. In this section the models used to describe the quenching bath, the heat transfer processes, metallurgical transformations and evolution of stresses, are presented. 


\subsection{Bath dynamics}

The behavior of the quenching bath is modeled by the drift-flux mixture-model for multiphase flows. In this model the fraction of vapor and liquid is described by their corresponding fractions $\alpha_{v}$ and $\alpha_{l}\left(=1-\alpha_{v}\right)$. The model solves the continuity, momentum and turbulence conservation equations for the mixture, continuity of the vapor phase and conservation of thermal energy of the liquid phase. The mixture properties may be volume weighted $\left(\alpha_{m}=\alpha_{l} \rho_{l}+\alpha_{v} \rho_{v}\right)$ or mass weighted $\left(\boldsymbol{v}_{m}=c_{l} \boldsymbol{v}_{l}+c_{v} \boldsymbol{v}_{v}\right)$, where $c_{i}=\alpha_{i} \rho_{i} / \rho_{m}$ is the mass fraction of the $i^{t h}$-phase ( $v$ or $l$ ). Details about the derivation of the multiphase model can be found in [11].

The system of partial differential equations (PDE's) used to represent the quenching bath is the following:

Conservation of total mass of the mixture:

$$
\frac{\partial \rho_{m}}{\partial t}+\nabla \cdot\left(\boldsymbol{v}_{m} \rho_{m}\right)=0
$$

Conservation of vapor mass:

$$
\frac{\partial\left(\alpha_{v} \rho_{v}\right)}{\partial t}+\nabla \cdot\left(\alpha_{v} \rho_{v} \boldsymbol{v}_{m}\right)=\Gamma_{v}-\nabla \cdot\left(\alpha_{v} \frac{\rho_{l} \rho_{v}}{\rho_{m}} \boldsymbol{V}_{v j}\right)
$$

Conservation of momentum of the mixture:

$$
\begin{aligned}
\frac{\partial\left(\rho_{m} \boldsymbol{v}_{m}\right)}{\partial t}+\nabla & \cdot\left(\rho_{m} \boldsymbol{v}_{m} \boldsymbol{v}_{m}\right)=-\nabla p_{m}+\rho_{m} \boldsymbol{g} \\
& +\nabla \cdot\left[\overline{\mathbb{T}}+\mathbb{T}^{T}-\nabla \cdot\left(\frac{\alpha_{v}}{\alpha_{l}} \frac{\rho_{l} \rho_{v}}{\rho_{m}} \boldsymbol{V}_{v j} \boldsymbol{V}_{v j}\right)\right]
\end{aligned}
$$

Conservation of thermal energy of the liquid:

$$
\frac{\partial\left(\alpha_{l} \rho_{l} c_{p, l} T_{l}\right)}{\partial t}+\nabla \cdot\left(\alpha_{l} \rho_{l} c_{p, l} T_{l} \boldsymbol{v}_{l}\right)=-\nabla \cdot \alpha_{l}\left(\boldsymbol{q}_{l}+\boldsymbol{q}_{l}^{T}\right)-\Gamma_{v} c_{p, l}\left(T_{s a t}-T_{l}\right)
$$

The model assumes that the vapor phase is at saturation temperature at every moment. Because of this assumption, only the thermal energy of the liquid phase has to be solved in eq. 4. In addition to the system $1-4$, a turbulence model to describe $\mathbb{T}^{T}$ is needed. Standard $k-\epsilon$ model with turbulent viscosity modified to take into account the presence of bubble-induced turbulence, as developed in [12, ch.12 sec.1.4], is used. The whole model is closed with the relationships of drift velocity, $V_{v j}=\sqrt{2 \sqrt{\sigma g \Delta \rho} / \rho_{l}}\left(1-\alpha_{v}\right)^{2}-D_{d}^{\alpha} \nabla \alpha_{v} / \alpha_{v}$ [12, ch.13 sec.1.2]. A condensation function $\Gamma_{v}=Q_{l v} /\left(c_{p, l}\left(T_{s a t}-T_{l}\right)\right)$, where the heat transfer function from the vapor to the liquid $\left(Q_{l v}\right)$ uses the Ranz-Marshall correlation under the premise that the continuous phase is the mixture [13].

Simultaneously to the evolution of the quenching bath, the temperature of the solid workpiece is described by:

$$
\frac{\partial\left(\rho_{s} c_{p, s} T_{s}\right)}{\partial t}-\nabla \cdot\left(k_{s} \nabla T_{s}\right)=0
$$


This equation is coupled to $(1-4)$ by the boundary conditions (BC's) that are imposed in the common boundaries between domains.

The BC's that have to be carefully modeled are: wall stress (eq. 6), heat flux delivered to the liquid (eq. 7), heat flux released from the solid (eq. 8) and injection of vapor due to vaporization (eq. 9). The BC's are described in general terms in eqs. 6 to 9 and fully detailed in following sections.

$$
\begin{aligned}
\left(\mu_{m}+\mu_{m}^{T}\right) \frac{\partial \boldsymbol{v}_{m}}{\partial n}=\tau_{w} \quad \text { and } \quad \boldsymbol{v}_{m} \cdot \boldsymbol{n}=0 \\
-\alpha_{l} k_{l} \frac{\partial T_{l}}{\partial n}=q_{w}^{l} \\
-k_{s} \frac{\partial T_{s}}{\partial n}=q_{w}^{s} \\
\alpha_{v} \rho_{v} \boldsymbol{v}_{v} \cdot \boldsymbol{n}=\dot{m}_{v}
\end{aligned}
$$

where $\boldsymbol{n}$ is the normal direction to the boundary.

The wall stress was modeled by the relationship $\tau_{w}=\rho_{m} \cdot\left(v_{m} / u^{+}\right)^{2}$, where $u^{+}$is an adequate wall-function, as is presented in [11]. The rest of boundary conditions are developed in further sections.

\subsection{Heat transfer}

The used heat transfer model is based on the partition of the total heat flux that is released from the solid surface, into specific contributions that depend on the physical phenomena that are modeled.

The vaporization dynamics presented here assumes two regimes clearly separated by the critical temperature. At temperatures higher than the critical one, it is assumed that the surface of the solid is covered by a vapor blanket. This stage produces moderate cooling rates due to the insulating effect of the vapor and does not inject remarkable amounts of it into the liquid. At temperatures below the critical one, the vapor blanket collapses and a burst of vapor is produced. Heat transfer rates are enormous at this point and only moderated by the amount of available liquid. All these physical aspects are recovered in the boundary conditions presented here.

As a first step, it is interesting to assess the heat flux that receives the liquid ( $q_{w}^{l}$, eq. 10) because this contribution will be present as a whole in the solid counterpart. The heat flux that receives the liquid is composed by two contributions: single-phase conductive heat flux $\left(f_{T} q_{\text {cond }}\right)$ and a radiative part $\left(q_{\text {rad }}\right)$.

$$
q_{w}^{l}=f_{T} q_{c o n d}+q_{\text {rad }}
$$

The conductive part contains a damping function $\left(f_{T}\right)$ that depends on wall temperature, and the properly named convective heat flux (eq. 11). This expression is based on the usual wall-law approach $T^{+}$. For details about the thermal wall-law, see ref. [11].

$$
q_{\text {cond }}=\alpha_{l} \frac{\left(T_{s}-T_{l}\right) \rho_{l} c_{p, l}}{T^{+}} \cdot \frac{v_{m}}{u^{+}}
$$


The radiative component corresponds to the model of radiation through a gray medium, whose expression is $q_{r a d}=\left(\epsilon_{0} \epsilon_{w}\right) /\left(\epsilon_{0}+\epsilon_{w}-\epsilon_{0} \epsilon_{w}\right) \sigma_{S B}\left(T_{s}^{4}-T_{l}^{4}\right)$, with $\epsilon_{0}=0.95$ and $\epsilon_{w}=0.6$ for the emissivities.

The total heat flux that is released from the solid (eq. 12) has two main components. A part dedicated to evaporate the liquid $\left(q_{\text {evap }}\right)$, and another to increase the liquid temperature. The latter was already presented in eq. 10 .

$$
q_{w}^{s}=-q_{\text {evap }}-q_{w}^{l}
$$

The evaporative part is conformed by three components:

$$
q_{\text {evap }}=\left(1-f_{T}\right) q_{f i l m}+f_{v} f_{T} q_{\text {nucl }}+q_{\text {sens }}
$$

where $q_{\text {film }}$ corresponds to the film boiling heat transfer mechanism, $q_{\text {nucl }}$ represents the nucleate boiling phenomenon and $q_{\text {sens }}$ is the released part that is necessary to raise the temperature of the liquid that is going to be evaporated up to the saturation temperature (sensible heating). The first two mechanisms are moderated by damping functions that depend on wall temperature $\left(f_{T}\right)$ and vapor fraction $\left(f_{v}\right)$. The $f_{T}$ is in charge to activate/deactivate the very high temperature heat transfer mechanism $\left(f_{T} \rightarrow 0\right.$ if $T_{s}>T_{\text {crit }}$ and $f_{T} \rightarrow 1$ if $T_{s}<T_{\text {crit }}$ ). At wall temperatures higher than a critical temperature $\left(T_{\text {crit }}\right)$, the dominant mechanism is film boiling, while nucleate and conductive parts are not active. The critical temperature is a function of liquid and solid properties and represents the temperature at which vapor blanket collapses, as it was studied and developed by Henry as presented in [14]. For the case studied here, the $T_{\text {crit }}$ is approximately constant and close to $525^{\circ} \mathrm{C}$, which is the value used along this work.

The $f_{v}$ damping function moderates the boiling mechanisms when a high vapor fraction accumulates near the surface of the piece. This function does not replicate the film boiling phenomenon and its consequent vapor blanket effect, but damps boiling if less liquid is available near the piece surface. Its functional form is similar to the one used by Končar in [13].

The film boiling mechanism is described by the correlation proposed by Bolukbasi [15] for vertical cylinders.

$$
q_{\text {film }}=0.0027\left(g\left(\rho_{l}-\rho_{v}\right) \Delta h / \nu_{v}\right)^{0.6} L_{c}^{0.8}\left(k_{v}\left(T_{s}-T_{\text {sat }}\right)\right)^{0.4}
$$

where $L_{c}$ a characteristic length, which in our case is taken as the diameter of the cylinder, $\nu_{v}$ is the kinematic viscosity of the vapor and $\Delta h$ is the difference of enthalpy between phases.

The nucleate boiling stage is modeled through the well known approach based on a frequency of departure for bubbles $\left(f_{b}\right)$, a number of active sites for nucleation of them $\left(N_{a}\right)$ and the amount of energy to evaporate a bubble of diameter $D_{d}$. Details about each correlation can be found in refs. [11, 16, 17]

$$
q_{n u c l}=f_{b} N_{a} \frac{\pi D_{d}^{3}}{6} \rho_{v} h_{l v}
$$

The boiling model considers two different heat transfer mechanisms responsible for liquid-vapor phase change, one (eq. 14) at very high temperatures and other active up to saturation values (eq. 15). These two mechanisms need to be complemented by an extra part 
responsible to elevate the temperature of the liquid that is going to be evaporated up to the saturation value. This contribution of sensible heat released by the solid wall depends on the mass flux of generated vapor and is presented in eq. 16.

$$
q_{\text {sens }}=\dot{m}_{v} c_{p, l} \rho_{l}\left(T_{\text {sat }}-T_{l}\right)
$$

Consequently, the mass flux of vapor produced during evaporation is:

$$
\dot{m}_{v}=\left(\left(1-f_{T}\right) q_{f i l m}+f_{v} f_{T} q_{n u c l}\right) / h_{l v}
$$

\subsection{Metallurgical transformations}

Steel has stable or metastable structures depending on its current temperature and the cooling rates that was subjected to. These structures can be formed by one or more solid phases. In general terms, there are several phases created from a diffusion mechanism and others from displacive transformations (diffusionless). During a cooling process, the austenite is considered as a high temperature phase (hot), while ferrite, pearlite, bainite and martensite are cold phases.

The ferritic, pearlitic and bainitic transformations can be described by a differential equation such as [1]:

$$
\dot{\hat{z}}(t)=f(T, \dot{T}, \hat{z}, d) \frac{\left[T-M_{s}\right]^{+}}{T-M_{s}}
$$

where $\hat{z}=\left\{Z_{f}, Z_{p}, Z_{b}\right\}$ are the proportions of ferrite, pearlite and bainite respectively, $d$ is the austenite grain diameter, $M_{s}$ is the martensite start temperature and $[X]^{+}$is the positive part of X.

For the martensitic transformation, the Koïstinen-Marburger equation is used:

$$
Z_{m}=\left(1-Z_{f}-Z_{p}-Z_{b}\right)\left\{1-\exp \left(\beta\left[T-M_{s}\right]^{+}\right)\right\}
$$

where $\beta$ is a material parameter.

\subsection{Generation of residual stresses}

The basic mechanisms that cause residual stresses, distortion of shape and volume changes during heating and cooling can be attributed primarily to three causes [18]:

- Internal stresses that cause changes in geometry when they exceed the yield strength of material. The limit value is reduced with increasing temperature.

- The stress caused by the differential expansion due to thermal gradients. This stresses increase with the temperature gradients and cause plastic deformation when the yield strength is exceeded.

- Changes in volume due to the metallurgical transformations. These volume changes modifies the residual stresses system, that it may exceed the yield strength. 
The mechanical problem is defined by the decomposition of various models of strain mechanisms:

$$
\dot{\varepsilon}=\dot{\varepsilon}^{e}+\dot{\varepsilon}^{p}+\dot{\varepsilon}^{t h}+\dot{\varepsilon}^{p t}
$$

where $\varepsilon, \varepsilon^{e}, \varepsilon^{p}, \varepsilon^{t h}$ and $\varepsilon^{p t}$ are strain contributions respectively named as, total, elastic, plastic, thermal and transformation induced plastic.

Metallurgical transformations imply changes in the mechanical properties of material. This effect is more important in plastic features (such as yield strength) and less in the elastic ones (Young's modulus and Poisson ratio). Thus, the classical model is used to define the elastic strain:

$$
\sigma=\overline{\bar{C}}(T) \varepsilon^{e}
$$

where $\sigma$ is the stress tensor and $\overline{\bar{C}}(T)$ is the elasticity tensor, depending only on the temperature.

Regarding plastic strain (without considering viscous effects), a Von Mises criterion with linear isotropic hardening is used. The yield function and the flow rule are defined:

$$
\begin{gathered}
f=\sigma_{e q}-R(T, Z, r)-\sigma_{y}(T, Z) \leq 0 \\
\dot{\varepsilon}^{p}=\dot{\lambda} \frac{\partial f}{\partial \sigma}
\end{gathered}
$$

where $\sigma_{e q}$ is the Von Mises stress: $\sigma_{e q}=\sqrt{\frac{3}{2} \sigma^{D}: \sigma^{D}}, R(T, Z, r)$ is the hardening function of material, $\sigma_{y}(T, Z)$ is the initial yield strength, $\sigma^{D}$ is the deviatoric stress tensor, $T$ is the temperature, $Z$ are the phase proportions and $r$ is a variable related to restoration effects $[3,4,5,6]$ (the accumulated plastic strain does not characterize completely the hardening state of material due to the restoration associated to phase transformations).

Regarding the factor $\lambda$, the consistency equation is verified:

$$
\dot{\lambda} \begin{cases}=0 & \text { if } f<0 \\ \geq 0 & \text { if } f=0\end{cases}
$$

An important fact that must be taken into account is that the material contains a variable proportion of metallurgical phases during the analysis. As it was commented previously, metallurgical transformations greatly influence the plastic properties (hardening and yield strength). These are defined for the five phases of steel. Generally, a linear relationship is chosen for a cold phases mixture and a non-linear law for a cold-hot phase mixture.

$$
\begin{gathered}
\sigma_{y}=\left(1-f_{h}(z)\right) \sigma_{y}^{\gamma}+f_{h}(z) \sigma_{y}^{\alpha} \\
R=\left(1-f_{h}(z)\right) R_{\gamma}+f_{h}(z) R_{\alpha}
\end{gathered}
$$

where $z$ is the amount of transformed cold phases: $z=Z_{f}+Z_{p}+Z_{b}+Z_{m}, f_{h}(z)$ is the nonlinear mixture law, $\sigma_{y}^{\gamma}$ is the yield strength of austenite, $\sigma_{y}^{\alpha}$ is the equivalent yield strength of 
cold phases: $\sigma_{y}^{\alpha}=\left(\sum_{i=1}^{4} z_{i} \sigma_{y}^{\alpha_{i}}\right) / z, R_{\gamma}$ is the hardening of austenite and $R_{\alpha}$ is the average hardening of cold phases: $R_{\alpha}=\left(\sum_{i=1}^{4} z_{i} R_{\alpha_{i}}\right) / z$.

The next type of analyzed strain is the thermal source one. In general, it is assumed:

$$
\varepsilon^{t h}=\alpha\left(T-T_{r e f}\right) \overline{\bar{I}}
$$

However, the previous equation is not valid for a material containing several phases. Two thermal expansion coefficients are defined: one for ferritic, pearlitic, bainitic and martensitic structures $\left(\alpha_{f}\right)$ and other for the austenite $\left(\alpha_{\gamma}\right)$.

A reference state where the thermal strain is null is also defined: a reference metallurgical phase (cold or hot) and a reference temperature $\left(T_{r e f}\right)$ are chosen. The reference phase is modeled by a function $\left(Z_{\gamma}^{R}\right)$ that it is 1 if the reference phase is the austenite and 0 if it is the cold phase.

Thermal strain for the austenite and the cold phases is written, respectively, by:

$$
\begin{gathered}
\varepsilon_{\gamma}^{t h}=\alpha_{\gamma}(T)\left(T-T_{r e f}\right) I-\left(1-Z_{\gamma}^{R}\right) \Delta \varepsilon_{f \gamma}^{T_{r e f}} I \\
\varepsilon_{f}^{t h}=\alpha_{f}(T)\left(T-T_{r e f}\right) I+Z_{\gamma}^{R} \Delta \varepsilon_{f \gamma}^{T_{r e f}} I
\end{gathered}
$$

where $T_{r e f}$ is the reference temperature, $\alpha_{\gamma}(T)$ is the average thermal expansion coefficient of austenite at current temperature (referred to the reference temperature), $\alpha_{f}(T)$ is the average thermal expansion coefficient of cold phases at current temperature (with respect to the reference temperature), $Z_{\gamma}^{R}$ is the function which defines the reference metallurgical phase and $\Delta \varepsilon_{f \gamma}^{T_{r e f}}$ reflects the difference in compaction between the crystallographic structures of austenite (FCC) and cold phases (BCC, BCT) at the reference temperature: $\Delta \varepsilon_{f \gamma}^{T_{r e f}}=\varepsilon_{f}^{t h}\left(T_{r e f}\right)-\varepsilon_{\gamma}^{t h}\left(T_{r e f}\right)$.

The thermal strain of a mixture of metallographical phases is defined by a linear law $\left(Z_{1}, Z_{2}, Z_{3}\right.$ and $Z_{4}$ are the proportions of ferrite, pearlite, bainite and martensite respectively):

$$
\begin{aligned}
\varepsilon^{t h}=\left(1-Z_{f}-Z_{p}-Z_{b}-Z_{m}\right) & {\left[\alpha_{\gamma}\left(T-T_{r e f}\right) I-\left(1-Z_{\gamma}^{R}\right) \Delta \varepsilon_{f \gamma}^{T_{r e f}} I\right] } \\
+ & \left(Z_{f}+Z_{p}+Z_{b}+Z_{m}\right)\left[\alpha_{f}\left(T-T_{r e f}\right) I+Z_{\gamma}^{R} \Delta \varepsilon_{f \gamma}^{T_{r e f}} I\right]
\end{aligned}
$$

Finally, the last analyzed strain mechanism is the transformation plasticity. Experimentally it is found that the dilatometric test of a specimen during the structural transformation is strongly influenced by the stress state and the application of loads, even below the yield strength of the material, can cause permanent deformation. This phenomenon will occur in steels subjected to thermal cycles including metallurgical transformations. It occurs during ferritic, pearlitic, bainitic and martensitic transformations, but not in the austenitic transformation.

The model for the transformation induced plasticity was proposed by Leblond $[3,4$, $5,6]:$

$$
\dot{\varepsilon}^{p t}=\frac{3}{2} \sigma^{D} \sum_{i=1}^{4} K_{i} F_{i}^{\prime}\left(1-Z_{\gamma}\right)\left\langle\dot{Z}_{i}\right\rangle
$$


where $\sigma^{D}$ is the deviatoric stress tensor, $Z_{\gamma}$ is the proportion of austenite, $\langle X\rangle$ is the positive part of X, $\dot{Z}_{i}$ are the transformation rates of cold phases, $K_{i}$ are constants, features of four cold phases and $F_{i}^{\prime}$ are functions of amount of transformed cold phase.

\section{NUMERICAL IMPLEMENTATION}

The problem presented here involves several coupled physical models of very different nature and mathematical behavior. Nevertheless, at leading order, the heat transfer model is decoupled from the metallurgical and mechanical model. This decoupled approach implies the assumption of adiabatic metallurgical transformations, and negligible mechanical effects (thermal dissipation and geometrical deformations) on heat transfer. Thus, the resolution of the whole problem can be done in two steps. The heat transfer model including the resolution of the two-phase flow of the bath and cooling of the workpiece is solved first. Then, using the evolution of the temperature in the workpiece, the transformation of metallurgical phases and development of stresses are solved separately.

Once the thermal transfer problems has been solved (along the considered time interval), instead of storing the temperature field history in the whole workpiece, only the temperature on the boundary is stored. This approach allows the use of different meshes for both subproblems (the thermal transfer problem and the metallurgical-mechanical problem), saves the interpolation operation of data from one mesh to the other and reduces the amount of data that has to be transfered from one subproblem to the other. As a disadvantage, the thermal problem in the workpiece is solved twice, but this small overload is completely affordable in the 2-D axisymmetric problem developed in section 4.

For the resolution of the heat transfer model Comsol Multiphysics ${ }^{1}$ software, version 3.5a, was used, while the metallurgical-mechanical problem was solved using Code_Aster ${ }^{2}$ version 11.0 code (a free software developed by Electricité de France). Both softwares are based on the finite element (FE) method. Comsol Multiphysics is a general purpose FE tool suitable to solve diverse physical problems. The 3.X version allows a flexible modification/inclusion of PDE's and BC's, which is desirable when non-standard problems, such as our thermal multiphase flow, are aimed to be solved. Code_Aster is able to resolve a wide variety of thermo-mechanical problems, including or not phase transformations. The selection this software is based on that our metallurgical-stress model is already implemented on it.

\subsection{Heat transfer}

Comsol Multiphysics have several built-in application modules to solve a wide variety of physical problems, in particular, the conduction-convection heat transfer (chcc) and mixture-model multiphase turbulent flows ( $\mathrm{chmm}$ ) modules are of our interest. Two different chcc modules are used for the workpiece and liquid temperatures. The mass transfer $\left(\Gamma_{v}\right)$ and the drift velocity $\left(\boldsymbol{V}_{v j}\right)$ are user-defined according to equations in section 2.2. The only relevant modification is the internal definition of the turbulent viscosity, where the effect of second phases is included according to [12].

\footnotetext{
${ }^{1}$ Comsol Multiphysics. http://www.comsol.com

${ }^{2}$ Electricité de France. http://www.code-aster.org
} 
The evolution problem is integrated using a Backward Differentiation Formula (BDF) of order 1 (Euler implicit) with adaptive time step. At each time step, the resulting nonlinear problem is solved using a fixed point technique where the complete thermal problem is segregated into five subproblems corresponding to:

1. mass and momentum conservation in the mixture: Eqs. 1 and 3 in $\boldsymbol{v}_{m}$ and $p$

2. vapor conservation: Eq. 2 in $\alpha_{v}$

3. turbulence model: standard $k-\epsilon$

4. thermal problem in the liquid: Eq. 4 in $T_{l}$

5. thermal problem in the workpiece: Eq. 5 in $T_{s}$

This approach allows to apply the present methodology to complex geometries (where a large mesh is expected to be needed, and a non-segregated algorithm would need to build a large stiffness matrix) even with moderate computational resources. In turn, each subproblem is solved using a damped Newton method (where a not-so-large tangent stiffness matrix must be computed and allowing the use of a direct, multifrontal method implemented in UMFPACK to solve the resulting linear systems) providing a good convergence rate.

The spatial domain is discretized using triangular elements, with the following selection of finite element basis:

- $P_{2}-P_{1}$ Taylor-Hood elements for $\boldsymbol{v}_{m}$ and $p$.

- $P_{1}$ elements for $\alpha_{v}$.

- $P_{2}$ elements for $T_{s}, T_{l}, k \& \epsilon$.

The convective terms in the previous equations are stabilized by means of SUPG artificial diffusion.

To adapt the time step, absolute and relative tolerances are set as 0.001 and 0.01 respectively. The maximum element size in the fluid domain is $5 \times 10^{-3}[\mathrm{~m}]$, while for the solid-fluid boundaries the maximum size is $5 \times 10^{-4}[\mathrm{~m}]$. The mesh consists of approximately 11000 triangular elements with a minimum quality of 0.8 . The spatial discretization gives a total of 105500 degrees of freedom to be solved. A desktop computer having a 4-core microprocessor Core $2 \mathrm{Q} 9400$ at $2.67 \mathrm{GHz}$ and 8 GB of RAM was used to solve the multiphase heat transfer problem. The calculation time took approximately 180 minutes for a 120 seconds span.

\subsection{Phases distribution and residual stresses}

The thermo-metallurgical and mechanical problem is, in turn, solved in sequential form. As explained above, the temperature history on the workpiece boundary is used to determine the temperature field by solving the corresponding thermal problem. In a second step, the computed temperature field history is used to determine the evolution of the metallurgical phase distribution in the whole workpiece (assuming that all the metallurgical transformations are, at leading order, independent on the stress field). Finally, both input fields (temperature 
and metallurgical phase distribution) are used to solve the mechanical problem in order to compute the geometrical distortions and residual stresses. Some details on the solution of each subproblem are given below.

The thermal transfer evolution problem in the workpiece is integrated used the implicit Euler algorithm with a fixed (and small) time step. At each time step, the resulting nonlinear problem (material parameters such as thermal conductivity, specific heat and density are dependent on the temperature) is solved using the Newton method. The spatial discretization is performed using quadrilateral $Q_{2}$-Serendipity elements. The quadrilateral mesh (that will be also used to solve the metallurgical transformation and mechanical problems) consists of around 7500 nodes.

Then the computation of the metallurgical transformation (given by 18-19) at the mesh nodes is performed. A method developed by Waeckel [1] is used to calculate the metallurgical phases distribution throughout the workpiece and at each instant of time. It is based on interpolation techniques and the fact that any experimentally known thermo-metallurgical curve (shown in a CCT diagram) is a particular solution of the differential equation of evolution. The CCT diagram of the material used in the analysis must be digitalized: start and finish temperatures of transformation according to the cooling curves, as well as the proportions of each phase at the end.

Finally, the mechanical problem must be solved to obtain the distortions and the residual stresses at the end of the quenching. The incremental, rate-independent plasticity problem is integrated using a loading curve (corresponding to the mechanical loads induced by the thermal gradients and the metallurgical phase transformations) divided according to the time step used in the integration of the thermal problem. Each loading step is solved using the Newton method with a line search (the stiffness matrix is recomputed every five iterations, and a direct multifrontal method is used to solve the resulting linear systems). The displacement field is discretized using quadrilateral $Q_{2}$-Serendipity elements, and the integration of the elasticplastic constitutive law (at each quadrature node) is performed using a radial return algorithm (the material parameters are dependent on the local metallurgical phase distribution). The same mesh used in the thermal problem is taken here.

A simulation of $120 \mathrm{~s}$ with a fixed time step $(0.1 \mathrm{~s})$ is made. Relative tolerances of $10^{-6}$ for the thermal problem and $5 \times 10^{-6}$ for the mechanical one were adopted. A desktop computer having an 8-core $i 7$ microprocessor at $2.67 \mathrm{GHz}$ with $12 \mathrm{~GB}$ of RAM was used. The calculation time for thermal, metallurgical and mechanical problems were approximately 8,2 and 180 minutes respectively.

\section{RESULTS}

As a workpiece of interest, a cylinder of $6.25 \mathrm{~mm}$ of radius and $60 \mathrm{~mm}$ length, immersed in a volume of $1.5 \mathrm{~L}$ of quenching oil (cylinder of $5 \mathrm{~cm}$ radius and $13 \mathrm{~cm}$ height), is considered. Due to the symmetry of the problem, a 2D-axisymmetric simplification is applied. The quenching bath is modeled having a forced velocity of $1 \mathrm{~m} / \mathrm{s}$ injected through the bottom of the fluid domain and an outlet through the upper side. The initial temperatures of the workpiece and fluid are $850^{\circ} \mathrm{C}$ and $60^{\circ} \mathrm{C}$ respectively. The evolution of temperatures (liquid and solid), vapor fraction, metallurgical phases and stresses is solved for a 120 seconds interval. 


\subsection{Heat transfer and cooling}

In order to feed the multiphase model, several physical properties of the oil and its vapor are needed. The most relevant ones are summarized in Table 1.

Table 1. Oil and vapor properties

\begin{tabular}{llll}
\hline & Liquid & & Vapor \\
\hline$\rho\left[\mathrm{kg} / \mathrm{m}^{3}\right]$ & 870 & & 3 \\
\hline$\mu[\mathrm{kg} /(\mathrm{m} . \mathrm{s})]$ & $9.40 \times 10^{-2}$ & $\left(20^{\circ} \mathrm{C}\right)$ & $2.3 \times 10^{-5}$ \\
& $2.35 \times 10^{-2}$ & $\left(40^{\circ} \mathrm{C}\right)$ & - \\
& $4.35 \times 10^{-3}$ & $\left(100^{\circ} \mathrm{C}\right)$ & - \\
& $1.00 \times 10^{-4}$ & $\left(217^{\circ} \mathrm{C}\right)$ & - \\
\hline $\mathrm{k}\left[\mathrm{W} /\left(\mathrm{m} .{ }^{\circ} \mathrm{C}\right)\right]$ & 0.14 & & 0.014 \\
\hline $\mathrm{c}_{p}\left[\mathrm{~J} /\left(\mathrm{kg} .{ }^{\circ} \mathrm{C}\right)\right]$ & 1800 & $\left(17^{\circ} \mathrm{C}\right)$ & 3000 \\
& 2300 & $\left(147^{\circ} \mathrm{C}\right)$ & - \\
\hline
\end{tabular}

In addition to these properties, a characteristic bubble diameter $\left(D_{b}\right)$ of $10^{-3}[\mathrm{~m}]$, a saturation temperature $\left(T_{s a t}\right)$ of $183\left[{ }^{\circ} \mathrm{C}\right]$, a latent heat of evaporation $\left(h_{l v}\right)$ of $10^{6}[\mathrm{~J} / \mathrm{kg}]$ and a vapor-liquid surface tension $(\sigma)$ of $3 \times 10^{-2}[\mathrm{~N} / \mathrm{m}]$ corresponding to a typical mineral oil used in quenching baths are assumed.

In Fig. 1 the evolution of vapor fraction around the cylinder and the solid temperature are shown for three representative instants. The transition of boiling mechanisms is depicted by the vapor fraction along the surface. At early stages, the whole surface is covered by a vapor blanket. This stable blanket produces a relatively mild heat exchange and therefore the net injection of vapor to the domain is also mild. Once the surface temperature of the solid goes below the critical value (see section 2.2), a switch from film to nucleate boiling modes happens. Nucleate boiling is characterized by very high heat transfer rates, and consequently, a very noticeable injection of vapor to the media. Both mechanisms and its transition can be seen in Fig. 1.
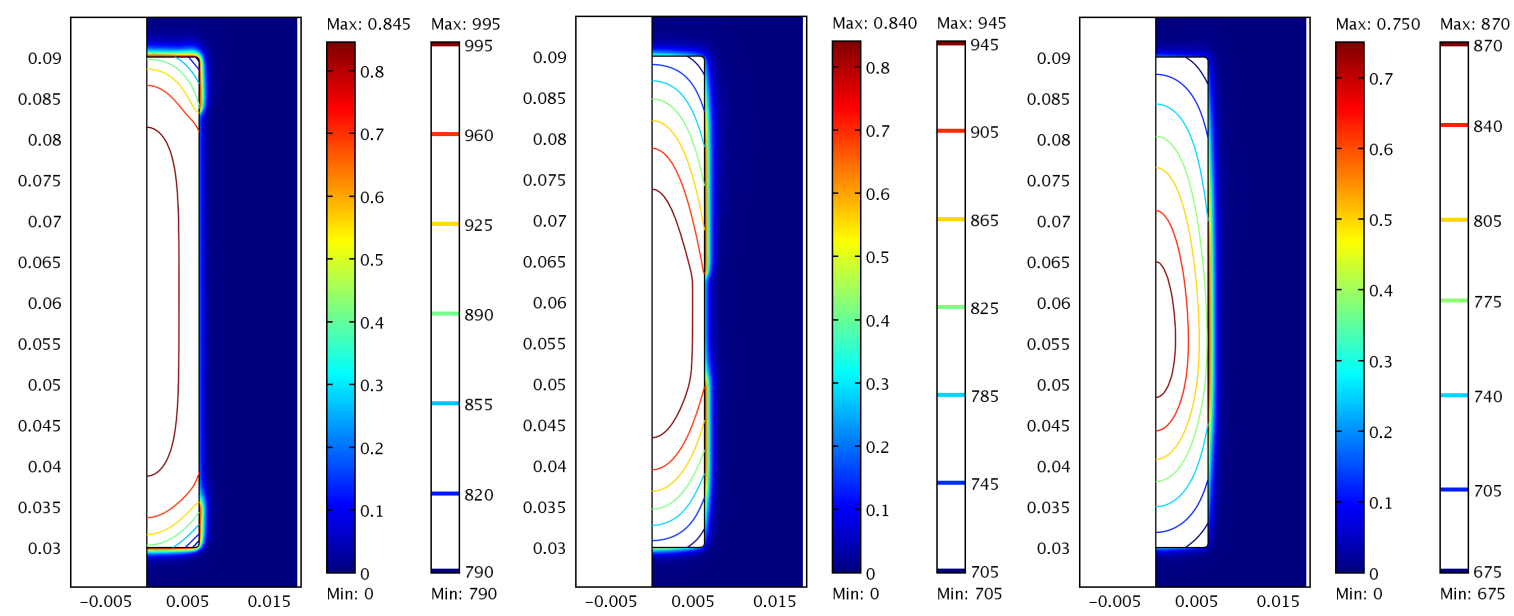

Figure 1. Time evolution of solid temperature and vapor fraction. From left to right: 6,8 and 9.5 seconds

This multiphase model for steel quenching produces heat transfer rates that are dependent not only on wall temperature, but also on others variables such as local flow velocity, 
vapor fraction and fluid temperature. In order to assess the variations that can be obtained by adopting this model, numerical results are compared against a typical heat transfer coefficient dependent only on wall temperature $\mathrm{h}\left(\mathrm{T}_{w}\right)$ obtained from a Jominy test with oil [9]. A comparison of heat transfer coefficients from each approach $\left(h=q_{w} /\left(T_{w}-T_{l}\right)\right.$ for numerical results) is presented in Fig. 2. Three positions along the longitude of the cylinder are analyzed, being $15 \%, 50 \%$ and $85 \%$ of the total height $\left(\mathrm{z}_{\text {Total }}\right)$. It is observed that numerical results and the correlation are in the same order of magnitude, although the temperature range where the predicted enhancement of heat transfer occurs is narrower than the correlation. The predicted heat transfer coefficient depends not only on wall temperature but also surface position, as it is expected.

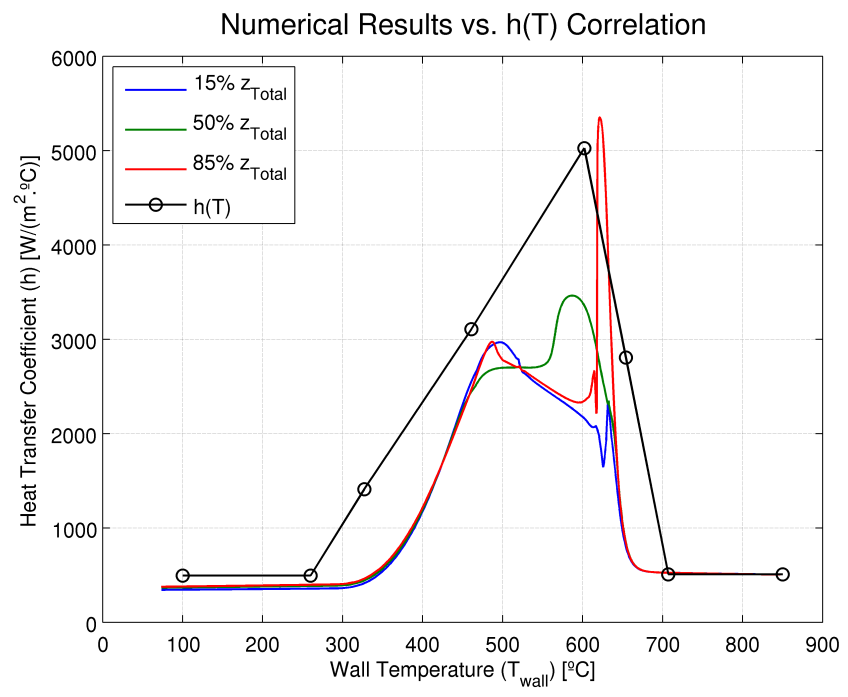

Figure 2. Heat transfer coefficients numerically obtained at different heights $\left(\mathrm{z}_{\text {Total }}\right)$ on the cylinder versus a typical $\mathrm{h}\left(\mathrm{T}_{w}\right)$ correlation

\subsection{Case 1 - Alloy Steel}

According to the detailed mechanisms in Section 2.4, a lot of material mechanical parameters must be defined by the user: Young's modulus $(E)$, Poisson ratio $(\mu)$, yield strength $\left(\sigma_{y}\right)$, modulus of hardening $(H)$ and thermal expansion coefficient $(\alpha)$. Thermal properties are also necessary (see Table 2 [18] [19]). In order to define properly the mechanical problem, null displacement at the center of the workpiece is assumed.

Table 2. Thermal properties of steel

\begin{tabular}{lll}
\hline Temp. $\left[{ }^{\circ} \mathrm{C}\right]$ & $\mathrm{k}\left[\mathrm{W} / \mathrm{m} .{ }^{\circ} \mathrm{C}\right]$ & $\rho c_{p}\left[\mathrm{~J} / \mathrm{m}^{3} .{ }^{\circ} \mathrm{C}\right]$ \\
\hline 20 & 37.7 & $3.46 \mathrm{e} 6$ \\
200 & 40.5 & $4.09 \mathrm{e} 6$ \\
300 & 39.5 & $4.42 \mathrm{e} 6$ \\
400 & 37.7 & $4.8 \mathrm{e} 6$ \\
600 & 33 & $5.0 \mathrm{e} 6$ \\
750 & 29.3 & $5.15 \mathrm{e} 6$ \\
900 & 25.3 & $5.15 \mathrm{e} 6$ \\
1000 & 26.9 & $5.2 \mathrm{e} 6$ \\
\hline
\end{tabular}


Table 3. Mechanical properties of alloy steel

\begin{tabular}{llll}
\hline Temp. $\left[{ }^{\circ} \mathrm{C}\right]$ & $\mathrm{E}[\mathrm{GPa}]$ & \multicolumn{2}{c}{$\sigma_{y}[\mathrm{MPa}]$} \\
& & Mart. & Aust. \\
\hline 20 & 208 & 1200 & 140 \\
100 & 204 & 1170 & 130 \\
200 & 200 & 1100 & 120 \\
400 & 180 & 980 & 110 \\
600 & 135 & 680 & 100 \\
700 & 80 & 350 & 70 \\
800 & 50 & 100 & 60 \\
900 & 32 & 50 & 30 \\
1000 & 30 & 20 & 20 \\
\hline
\end{tabular}

Table 3 shows some mechanical properties for the alloy steel. In addtition, $\alpha_{\text {Mart }}=$ $15\left[\mu \mathrm{m} / \mathrm{m} .{ }^{\circ} \mathrm{C}\right], \alpha_{\text {Aust. }}=23.5\left[\mu \mathrm{m} / \mathrm{m} .{ }^{\circ} \mathrm{C}\right]$ and Poisson's ratio $(\mu)$ constant with temperature and equal to 0.3. $H$ for martensite is got (as the previous data) from [19], with $H_{\text {aust }}=$ $1 / 10 H_{\text {mart }}$. The parameters of transformation induced plasticity and restoration models and the mixture law for multiphasic material are obtained from [19] and [20].

The stresses generated in the quenching process are of thermal and metallurgical origin, both of great influence during process. Figure 3 shows the time evolution of several magnitudes for the two points evaluated (core point $(\mathrm{pC})$ and surface point $(\mathrm{pS})$ as indicated in Figure 4(a)), up to 20 seconds, hereupon the variation of the stresses suffers only slight modifications.
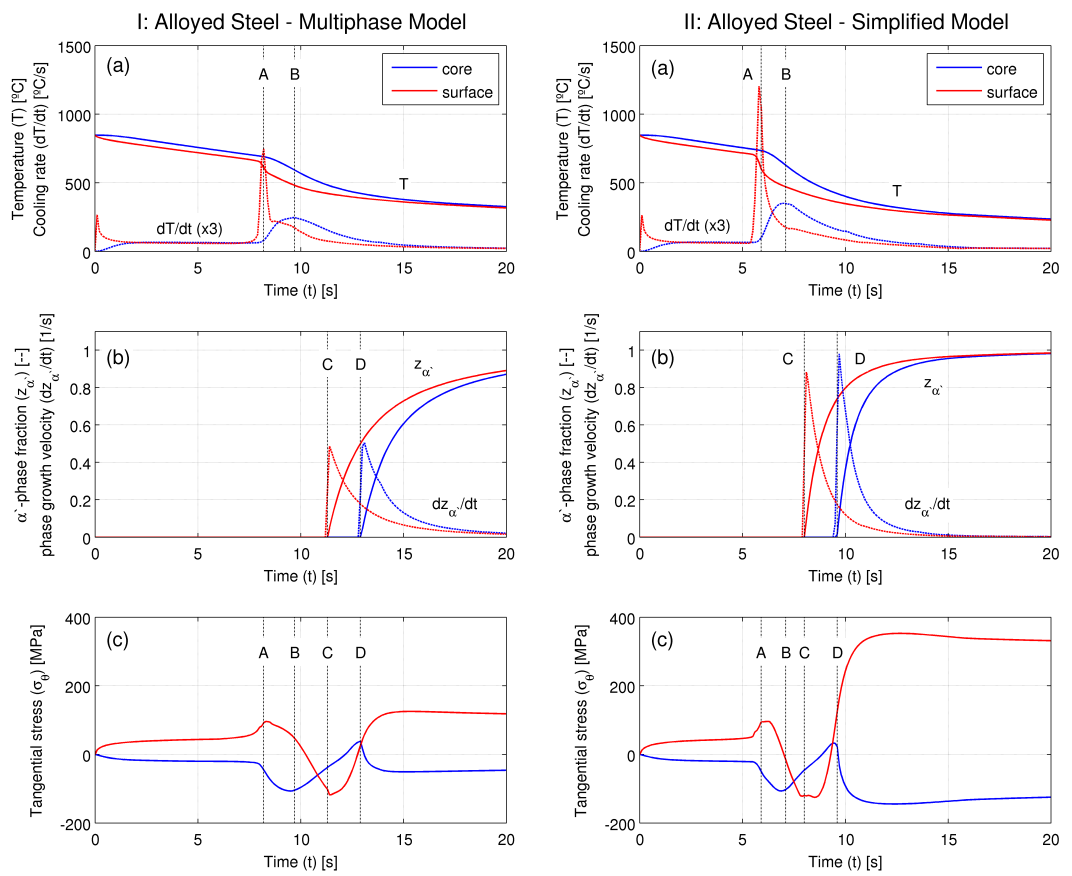

Figure 3. Alloy steel, I Multiphase Model, II Simplified Model. (a) Temperature and cooling rates, (b) phases evolution, (c) tangential stresses.

During the first stages internal stresses are only of thermal origin, for the reason that metallurgical transformation has not started (see first section of Figure 3(c) graphs, till time 
A), and increase until a local maximum is reached around time A. The specific volume difference between crust and core generates this stresses state, tensile in surface and compression inside [21, 22]. At this stage the cooling rates undergoes a sharp increase due to the change in the heat exchange mechanism between the workpiece and the fluid, causing an increase in the thermal gradient that raises the stresses value [18].

Once the point of greatest difference in temperature between the surface and core (point of intersection between the curves representing the cooling rate) is reached, the core is cooled (shrinks) faster than the surface, producing a reduction in the values of stresses [22]. However, the reduction of stresses can also be affected by the beginning of the transformation of the piece, which begins at the corners before it occurs in pS (Figure 4(c)). During martensitic transformation the specific volume is increased compared to austenitic phase, but the surrounding bears this increase by inducing compressive stresses in the transformed area.

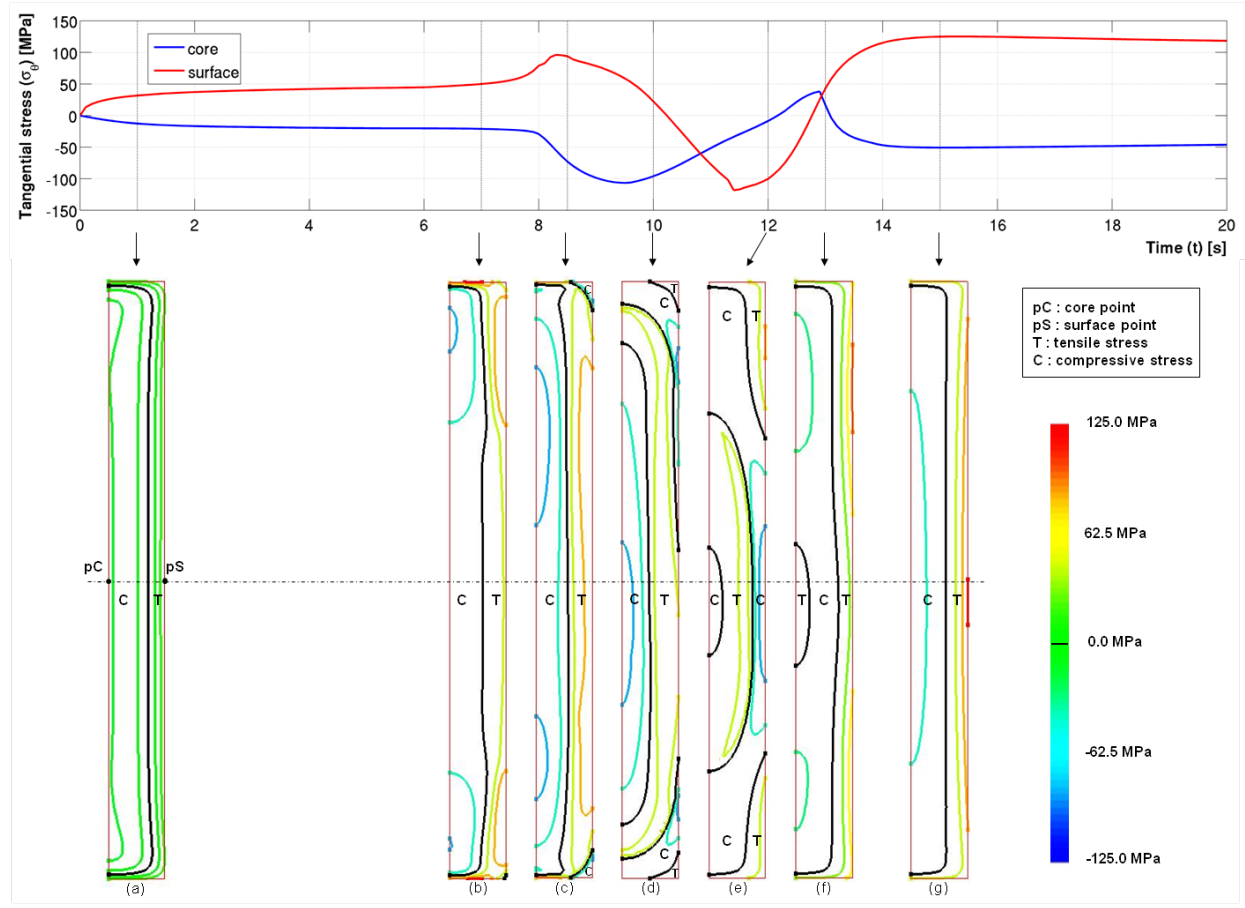

Figure 4. Evolution of stresses in the alloy steel, multiphase cooling model.

Metallurgical transformation begins at time $\mathrm{C}$ at the surface point $\mathrm{pS}$ evaluated, therefore, there is a change in the stresses value (Figure 3(c)) due to the volume increase. The final workpiece deformation in both modes can be described as barrel-shape. This form is associated with a quenching process where the greatest difference in temperature between the surface and core occurs before the phase transformation (Figure 5) [23]. This figure also includes the value of residual stresses at the piece surface and along the piece radius.

Differences that occur between the multiphase and the simplified cooling model can be seen in Figure 3(I (c) and II (c)). At the first stages, the two points analyzed are following the same temporal evolution, generating thermal stresses with similar values. Change in the cooling behavior occurs earlier in the simplified model, generating an advance in the beginning and growth rate of the martensitic phase. This transformation provokes the same change in the stresses evolution, just as in the full model, but the existence of higher temperatures and 


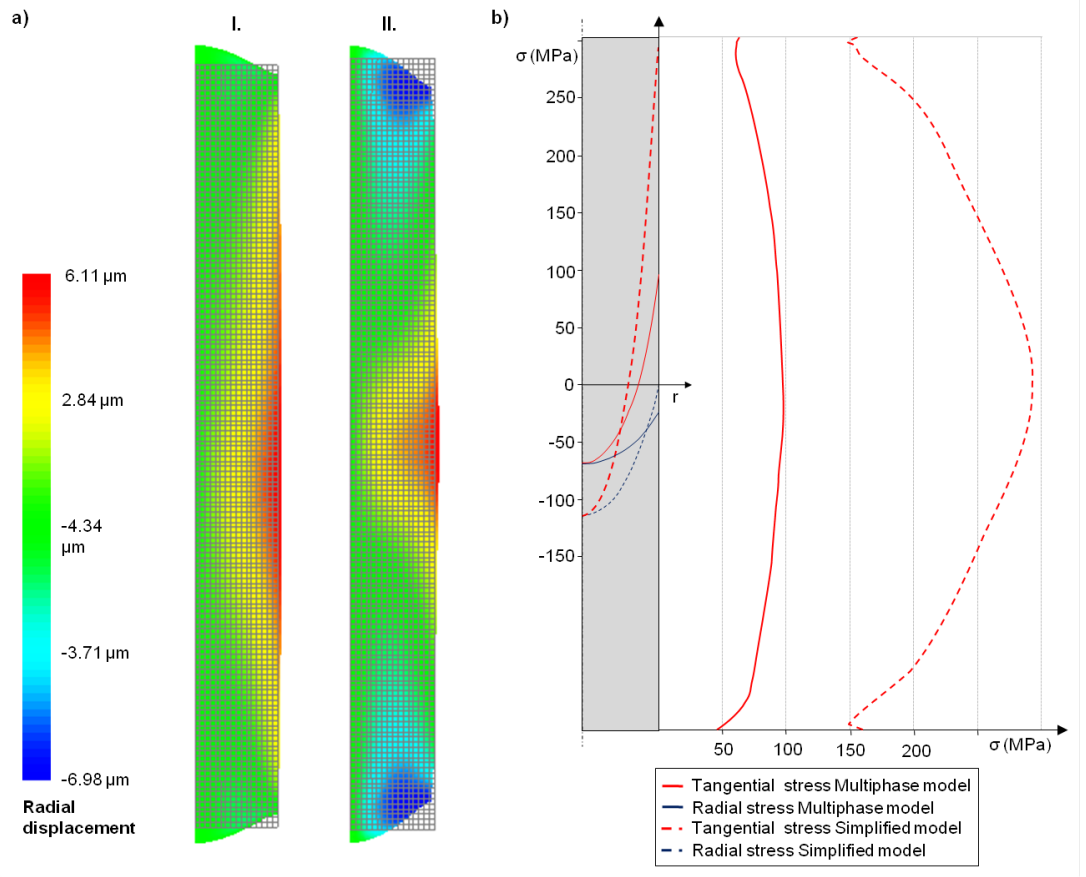

Figure 5. (a) Deformed shape and radial displacement, I. Multiphase model, II. Simple model. (b) residual stresses.

transformations gradients produces greater variation in residual stresses [18].

\subsection{Case 2 - Eutectoid Steel}

Table 4. Mechanical properties of eutectoid steel

\begin{tabular}{llllll}
\hline Temp. $\left[{ }^{\circ} \mathrm{C}\right]$ & $\mathrm{E}[\mathrm{GPa}]$ & $\mu[-]$ & \multicolumn{3}{c}{$\sigma_{y}[\mathrm{MPa}]$} \\
& & & Pearl. & Mart. & Aust. \\
\hline 0 & 205 & 0.28 & 450 & 1750 & 220 \\
300 & 185 & 0.3 & 230 & 1550 & 130 \\
600 & 165 & 0.31 & 140 & 1350 & 35 \\
900 & 124 & 0.35 & 30 & - & 35 \\
\hline
\end{tabular}

The mechanical properties for this material are obtained from [24]: Table 4, the thermal expansion coefficients of each phase $\left(\alpha_{\text {Mart. } / \text { Pearl }}=15\left[\mu \mathrm{m} / \mathrm{m} .{ }^{\circ} \mathrm{C}\right], \alpha_{\text {Aust. }}=21.7\right.$ $\left.\left[\mu \mathrm{m} / \mathrm{m} .{ }^{\circ} \mathrm{C}\right]\right)$, including the hardening modulus for each phase. A linear mixture law is assumed and the parameters of transformation induced plasticity and restoration models are the same as the alloy steel.

The stresses behavior of the eutectoid steel is slightly different than the alloy steel. Due to the relationship between chemical composition and the cooling rates, the appearance of pearlite occurs before the workpiece quenching starts. In Figures 6 and 7 it is showed only the interest stages of workpiece cooling. It is likely that the metallurgical calculation given by equation 18 could be improved for this case.

The stresses induced in the workpiece until time A (Figure 6(a)) are of thermal origin. However, from this time on, the stress state is influenced by thermal and metallurgical causes. The maximum tensile surface stresses match up with the start of pearlite transformation (time 

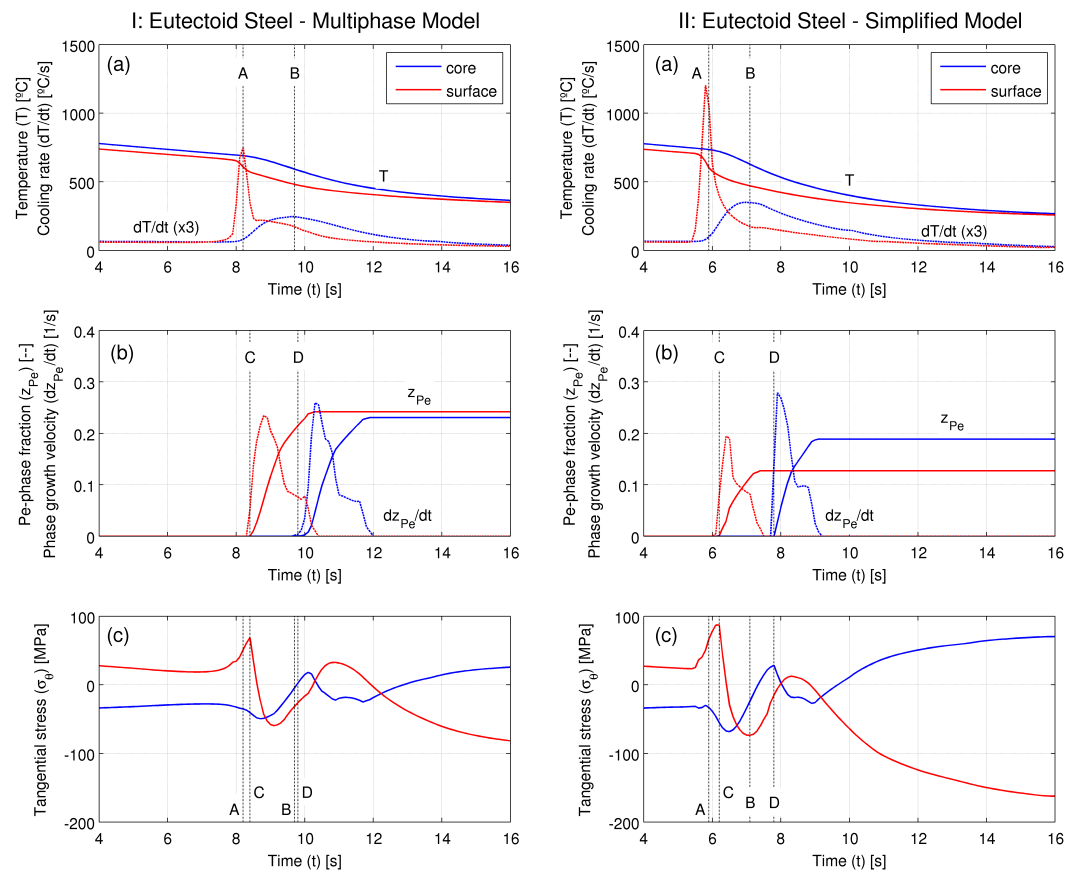

Figure 6. Eutectoid steel, I Multiphase Model, II Simplified Model. (a) Temperature and cooling rates, (b) phases evolution, (c) tangential stresses.

$\mathrm{C}$ and D, Figure 6 ), and the greater temperature difference occurs after the start of surface transformation, unlike it happens in the previous case. At this moment, the compressive stress in the core increases to balance the stress state, and the core cools faster. This has a great influence on the generated thermal stresses until the beginning of the transformation in the core [18]. Therefore, there is a combination of effects (thermal-metallurgical) inducing tension in the workpiece. This circumstance provokes a spool-shaped deformation as it is expected [23].
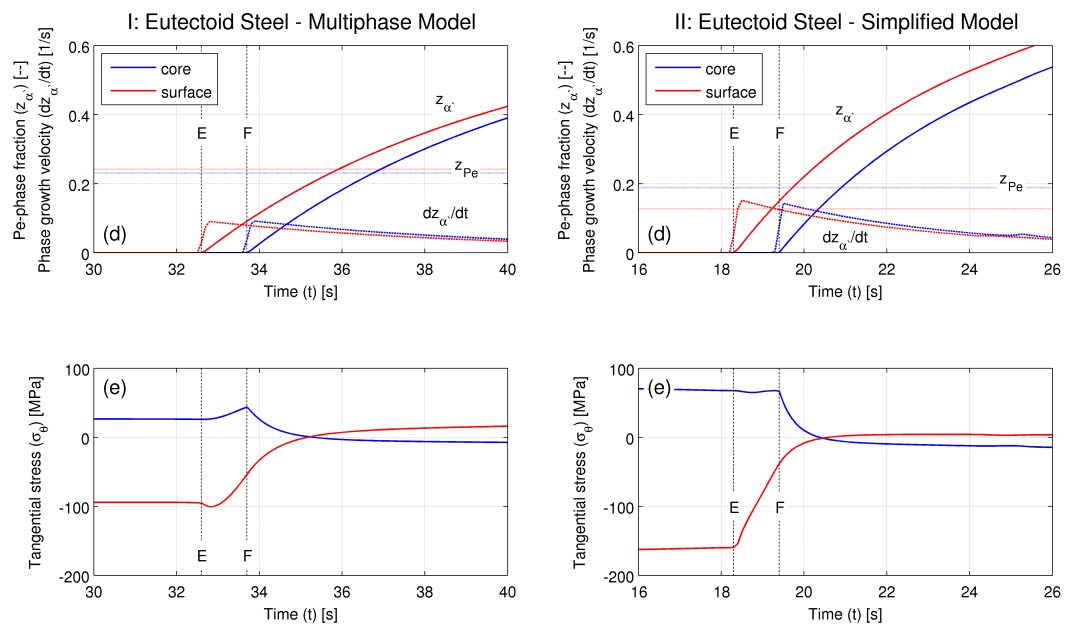

Figure 7. Quenching phase for eutectoid steel, I Multiphase Model, II Simplified Model. (d) Phases evolution, (e) tangential stresses. 
From the last time showed in Figure 6, thermal effects do not influenced and the temperature of the workpiece is homogenized. So, only stresses induced by metallurgical changes happened, starting with a martensitic transformation, and resulting in the same consequences as in the alloy steel (Figure 7). The stresses behavior that occur between the multiphase and the simple cooling model for the eutectoid steel are similar to those that occur in the alloy case. Higher cooling rates provoke that the transformation occurs before and stresses value are increased due to the greater thermal and composition gradients.

\section{CONCLUSIONS}

A novel heat transfer model for quenching based on the resolution of the multiphase liquid-vapor flow around the workpiece was applied to a very basic case of interest. The quenching process of a cylinder was modeled taking into account different conditions. Two different materials were considered, being those, a medium alloy steel and an eutectoid one. In addition, the effect of the heat transfer model on final properties was assessed by comparing results obtained by the multiphase model versus a model based on a simplified $\mathrm{h}\left(\mathrm{T}_{w}\right)$ correlation.

It was observed that the heat transfer coefficients obtained from the multiphase model are qualitatively and quantitatively similar to the $\mathrm{h}\left(\mathrm{T}_{w}\right)$ correlation considered. However, the multiphase model predicts a variation of the heat transfer coefficient along the surface. This feature breaks the longitudinal symmetry that is obtained by simpler approaches. It is expected a more dramatic effect of this feature if a workpiece of a more complex geometry is considered.

Even though the patterns of evolution of the residual stresses are similar, the predicted final stresses do have noticeable differences from one approach to another. This result is because the simplified approach produces faster cooling velocities, which will produce earlier and faster metallurgical transformations. The combination of faster cooling and transformations lead to a higher level of residual stresses and geometrical distortions if the simplified $\mathrm{h}\left(\mathrm{T}_{w}\right)$ approach is adopted.

As a final remark, the effect of different heat transfer models and its effect in the induced residual stresses after quenching was assessed. Even though both models have small differences in the effective heat transfer coefficient, the level of induced residual stresses can be noticeably different. Also differences along the geometry can be found. Due to these facts, it is very important to get a precise and accurate model to describe the quenching process if improvements on the prediction of metallurgical and mechanical problems are pursued.

\section{Acknowledgements}

This work was partially funded by project MTM2010-21135-C02-02 of the Spanish MEC and FEDER (EU), and projects IN845B-2010/046 \& 09DPI189E of Xunta de Galicia \& CIE Galfor S.A. (Spain). DNP acknowledges his PhD scholarship from MAEC-AECID (Spain). 


\section{REFERENCES}

\section{References}

[1] F. Waeckel. Une loi de comportement thermo-mtallurgique des aciers pour le calcul mcanique des structures. PhD thesis, Ensam Paris, 1994.

[2] Sabine Denis, Pierre Archambault, Elisabeth Gautier, André Simon, and Gérard Beck. Prediction of residual stress and distortion of ferrous and non-ferrous metals: Current status and future developments. Journal of Materials Engineering and Performance, 11:92-102, 2002. 10.1007/s11665-002-0014-2.

[3] J.B. Leblond, G. Mottet, and J.C. Devaux. A theoretical and numerical approach to the plastic behaviour of steels during phase transformationsi. derivation of general relations. Journal of the Mechanics and Physics of Solids, 34(4):395 - 409, 1986.

[4] J.B. Leblond, G. Mottet, and J.C. Devaux. A theoretical and numerical pproach to the plastic behaviour of steels during phase transformationsii. study of classical plasticity for ideal-plastic phases. Journal of the Mechanics and Physics of Solids, 34(4):411 432, 1986.

[5] J.B. Leblond, J. Devaux, and J.C. Devaux. Mathematical modelling of transformation plasticity in steels i: Case of ideal-plastic phases. International Journal of Plasticity, 5(6):551 - 572, 1989.

[6] J.B. Leblond. Mathematical modelling of transformation plasticity in steels ii: Coupling with strain hardening phenomena. International Journal of Plasticity, 5(6):573 - 591, 1989.

[7] M Eshraghi Kakhki, A Kermanpur, and M A Golozar. Numerical simulation of continuous cooling of a low alloy steel to predict microstructure and hardness. Modelling and Simulation in Materials Science and Engineering, 17(4):045007, 2009.

[8] Arif Sugianto, Michiharu Narazaki, Minoru Kogawara, Atsushi Shirayori, Soo-Young Kim, and Satoshi Kubota. Numerical simulation and experimental verification of carburizing-quenching process of scr420h steel helical gear. Journal of Materials Processing Technology, 209(7):3597-3609, 2009.

[9] B. Smoljan. Numerical simulation of steel quenching. Journal of Materials Engineering and Performance, 11:75-79, 2002. 10.1007/s11665-002-0011-5.

[10] Willem J. J. Vorster, Morne W. Van Der Watt, Andrew M. Venter, Edward C. Oliver, D. G. Leo Prakash, and Alexander M. Korsunsky. Influence of quenchant hydrodynamics and boiling phase incipient temperature shifts on residual stress formation. Heat Transfer Engineering, 30(7):564-573, 2009. 
[11] Diego N. Passarella, Elena B. Martín, and Fernando Varas. Mathematical modeling of heat transfer during quenching process. Conference on Numerical Methods for Hyperbolic Equations: Theory and Applications, 2012. Santiago de Compostela, July 4-8, 2011. Proceedings to be published.

[12] Mamoru Ishii and Takashi Hibiki. Thermo-fluid Dynamics of Two phase-flow. Springer, New York, 2006.

[13] Boštjan Končar and Iztok Tiselj. Influence of near-wall modelling on boiling flow simulation. Nuclear Engineering and Design, 240(2):275 - 283, 2010. Twelfth International Topical Meeting on Nuclear Reactor Thermal Hydraulics (NURETH-12).

[14] Roy Freud, Ronen Harari, and Eran Sher. Collapsing criteria for vapor film around solid spheres as a fundamental stage leading to vapor explosion. Nuclear Engineering and Design, 239(4):722-727, 2009.

[15] Abdurrahim Bolukbasi and Dogan Ciloglu. Investigation of heat transfer by means of pool film boiling on vertical cylinders in gravity. Heat and Mass Transfer, 44:141-148, 2007. 10.1007/s00231-007-0238-7.

[16] G. Kocamustafaogullari and M. Ishii. Interfacial area and nucleation site density in boiling systems. International Journal of Heat and Mass Transfer, 26(9):1377-1387, 1983.

[17] G. Kocamustafaogullari and M. Ishii. Foundation of the interfacial area transport equation and its closure relations. International Journal of Heat and Mass Transfer, 38(3):481-493, 1995.

[18] George E. Totten, Maurice A.H. Howes, and Tatsuo Inoue. Handbook of Residual Stress and Deformation of Steel. ASTM International, 2001.

[19] N.T. Trinh. Sur la Modélisation du Comportement Thermomécanique et Métallurgique des Aciers. PhD thesis, École Polytechnique, 2008.

[20] S. Petit-Grostabussiat. Conséquences mécaniques des transformations structurales dans les alliages ferreux. PhD thesis, Institut National des Sciences Appliquées de Lyon, 2000.

[21] T. Ericsson and B. Hildenwall. Thermal and transformation stresses. Sagamore Army Materials Research Conference Proceedings, 1982.

[22] C. Jensen. Dimensional changes during heat treatment of hot work tool steels. Industrial Heating, 60(3), 1993.

[23] H. Berns. Distortion and crack formation by heat treatment of tools. Veitsch-Radex Rundschau, (1), 1989.

[24] Caner Şimşir and C. Hakan Gür. 3d fem simulation of steel quenching and investigation of the effect of asymmetric geometry on residual stress distribution. Journal of Materials Processing Technology, 207(1-3):211-221, 2008. 